\title{
Facilitators and Barriers of External Coaches’ Involvement into School-Based Extracurricular Sports Activities: A Qualitative Study
}

\author{
Kenryu Aoyagi ${ }^{1}$, Kaori Ishii ${ }^{2}$, Ai Shibata ${ }^{2}$, Hirokazu Arai $^{3}$, Chisato Hibi ${ }^{1}$, Koichiro Oka ${ }^{2}$ \\ ${ }^{1}$ Graduate School of Sport Sciences, Waseda University, Saitama, Japan \\ ${ }^{2}$ Faculty of Sport Sciences, Waseda University, Saitama, Japan \\ ${ }^{3}$ Faculty of Letters, Hosei University, Tokyo, Japan \\ Email: ken-ryu.ao-yagi@ruri.waseda.jp
}

Received May $2^{\text {nd }}$, 2013; revised June $2^{\text {nd }}, 2013$; accepted June $9^{\text {th }}, 2013$

Copyright ( 2013 Kenryu Aoyagi et al. This is an open access article distributed under the Creative Commons Attribution License, which permits unrestricted use, distribution, and reproduction in any medium, provided the original work is properly cited.

\begin{abstract}
School-based extracurricular sports activity (SBECSA) provides one of the main opportunities for adolescents to play sports in Japan. However, maintaining active SBECSA is difficult because of the large burden on teachers to manage SBECSA and a lack of SBECSA teachers who can coach expertly. To resolve these issues, the recruitment of external coaches has been promoted. However, the number of coaches and frequency of coaching are not sufficient for the current demand. Additionally, it is not clear how to promote the engagement of external coaches. Therefore, the purpose of the present study was to explore a variety of facilitators and barriers associated with the involvement of external coaches into SBECSA. Twenty-five external coaches were recruited from junior high and high schools across Japan. Data obtained through personal semi-structured interviews were analyzed using the KJ method (a qualitative type of analyses). As a result, seven facilitators (positive feelings, adequate system, positive social support, comfortable climate of SBECSA, environment, growth of external coach, network building), and six barriers (negative feelings, inadequate system, lack of support, uncomfortable climate of SBECSA, poor environment, burdens) were identified. In conclusion, SBECSA would become more attractive for external coaches by enhancing facilitators and reducing barriers.
\end{abstract}

Keywords: Adolescent; After School; Coach; KJ Method; Volunteer

\section{Introduction}

Engagement in exercise and sports has been recommended for adolescents to prevent decreased physical fitness and to enhance healthy development (Ministry of Education, Culture, Sports, Science and Technology in Japan: MEXT, 2011). Similar to foreign countries such as Australia, Canada, and the UK (Sport Council Wales, 2009; Edwards, Kanters, \& Bocarro, 2011; Australian Bureau of Statistics, 2012), school-based extracurricular sports activity (SBECSA) provides one of the main opportunities to play sports for Japanese adolescents. Junior high and high school students play SBECSA under the supervision of teachers after school and on weekends. SBECSA includes sports such as baseball, soccer, basketball, track and field, swimming, and judo. According to the Course of Study (curriculum guide for defining basic standards for education) published by MEXT, schools should implement SBECSA to complement the educational curriculum as part of the school education. Therefore, SBECSA is strongly interconnected with school education and recognized as an extremely valuable opportunity (MEXT, 2008; MEXT, 2009a). In 2009, 64.9\% of junior high school students (75.5\% males and 53.8\% females) and $40.7 \%$ of high school students (54.5\% males and $26.6 \%$ females) in Japan participated in SBECSA (MEXT, 2009b).
SBECSA offers a number of positive benefits such as enjoyment or a purpose in life, building a solid foundation to enjoy sports throughout life, improving physical fitness and health, cultivating a rich humanity, and contributing to a bright and fulfilling school life (MEXT, 1997). A Japanese nation-wide survey for physical fitness revealed a positive relationship between participation in SBECSA and high physical fitness in adulthood (MEXT, 2012). Additionally, participation in SBECSA was positively correlated with academic performance (e.g. grade point average, math and science test scores) (Fredricks \& Eccles, 2006; Lipscomb, 2007), school bonding (Barnett, 2007; Dotterer, McHale, \& Crouter, 2007), psychological adjustment (e.g. depression, self-esteem, and concentration) (Fredricks \& Eccles, 2006; Shernoff \& Vandell, 2007), and friendship (Schaefer, Simpkins, Vest, \& Price, 2011). These findings from previous research studies suggest that SBECSA plays an important role in the healthy development of young people both short- and long-term.

However, there are issues that can negatively affect student participation in SBECSA and thus limit the acquisition of sports skills. Generally, full-time teachers coach SBECSA (Tokyo Metropolitan Board of Education, 2008), but sometimes they are assigned to coach sports activities that they cannot coach expertly (MEXT, 1997; MEXT, 2010). Previous studies 
demonstrated the importance of expert coaching for positive youth development (e.g. performance skill, confidence, positive social relationship, and morality) (Cote \& Gilbert, 2009; Stewart, Lindsay, \& Trevor, 2011). Considering these findings, some teachers cannot contribute to the improvement of performance skills or confidence of team members. Therefore, recruitment of an external coach who can coach expertly is valuable for team members' development. Moreover, there are physical, monetary, and mental challenges related to managing SBECSA (MEXT, 1997; Japan Senior High School Teachers and Staff Union, 2008; Whiteley \& Richard, 2012). In addition, inactivity or discontinuation of SBECSA sometimes occurs because a teacher is transferred to another school (School-based Extracurricular Sports Activity in Junior High School "Nagano Model" Exploratory Committee, 2004; Nakazawa, 2011). Public school teachers are generally required to transfer to another school once every several years in Japan. At that time, if there is no substitute teacher who can coach SBECSA, these activities are sometimes eliminated.

To resolve these issues, there has been a growing interest in promoting the involvement of external coaches. An external coach is defined as a person who coaches school-based extracurricular activity instead of, or as support for, the teacher (Sasakawa Sports Foundation: SSF, 2011). An external coach may be a part-time teacher, a sports club coach, leader of a social physical education program, graduate of the school in question, or a student's parent (All Japan High School Athletic Federation, 2012). There is no common rule on how to manage external coaches, and they are engaged in SBECSA with a wide range of compensation (from no compensation to compensation as a full-time job) (SSF, 2011). In Australia and the UK, extracurricular sports activities have been outsourced similar to that in Japan (Flintoff, 2008; Griggs, 2010; Williams, Hay, \& Macdonald, 2011).

However, some issues underlie the involvement of external coaches in SBECSA. A previous survey indicated that external coaches do not coach frequently enough (Miyagi Prefecture Board of Education, 2008; Yamagata Prefecture Board of Education, 2010). Additionally, other issues such as difficulty in securing human resources (Miyagi Prefecture Board of Education, 2008; Yamagata Prefecture Board of Education, 2010; Williams, Hay, \& Macdonald, 2011) and the small number of external coaches available by region and type of sport have been reported (Nishijima, Yano, \& Nakazawa, 2007; Nippon Junior High School Physical Culture Association, 2012). Thus, the lack of external coaches for SBECSA is problematic.

To promote the involvement of external coaches, it is important to enhance facilitators and reduce barriers that encourage or discourage external coaches' participation in SBECSA. Previous studies have attempted to clarify the benefits and burdens for external coaches in Japan (Shioya, 2002; Kanagawa Prefecture Board of Education, 2008; Miyagi Prefecture Board of Education, 2008). However, most studies were conducted using questionnaires with few question items and a focus on only one prefectural area. Although LaVoi and Dutove (2012) revealed barriers and supported for female coaches, most participating coaches were male and worked in universities as professional coaches. Thus, previous studies may only partially explain facilitators and barriers, and how to promote the engagement of external coaches into SBECSA is less clear. To explore factors that could comprehensively contribute to increased numbers and coaching frequency of external coaches, the use of a quail- tative method (i.e. interview) and collection of opinions of external coaches are necessary. Therefore, the purpose of the present study was to explore the facilitators and barriers associated with external coaches' involvement in SBECSA.

\section{Methods}

\section{Participants}

Participants comprised 25 external coaches who had coached at a public junior high school or a public high school in October 2010. They were introduced by connected teachers and had varying sociodemographics and characteristics of SBECSA including age, gender, occupation, type of school, prefecture, and type of sport coached. Participants were recruited from 13 prefectural areas and 15 different sports (archery, badminton, baseball, basketball, dance, handball, kendo, rubber-ball baseball, rugby, soccer, softball, soft tennis, table tennis, track and field, and volleyball). They were offered a gift card worth 1000 yen (8 US dollars, 7 pounds sterling or 8 Euros in October 2010) for participating in the interview. The research proposal was approved by the ethics board at Waseda University. All participants were informed of the purpose and design of the study, and written informed consent was obtained from each before enrollment.

\section{Interview Procedure}

First, sociodemographics and characteristics of SBECSA (e.g. type of school, prefecture, sport, coaching experience, and compensation) were obtained in writing from each participant. Second, a personal semi-structured interview was conducted following a pre-determined interview guide. The interview guide was developed through pilot interviews using five external coaches. According to feedback from the coaches, confusing questions were modified and the interview skills of the interviewer were developed. The question items included two open-ended questions as follows: 1) What are facilitators of involvement in SBECSA? 2) What are barriers to involvement in SBECSA? Participants were asked to respond freely to the questions, and all topics were explored until exhausted. Each interview took between $20-60$ minutes (mean $=33.8$ minutes). Interviews were performed from December 2010-March 2011 at a place convenient for each participant such as university, community center, or school where the external coach was involved. All interviews were conducted by a single researcher and audio recorded with each participant's agreement.

\section{Analysis}

Each recorded interview was transcribed verbatim. The KJ method (Kawakita, 2004) was used to analyze the transcribed data. The KJ method is a qualitative analysis that contains abductive procedures such as label making and label grouping. Therefore, the KJ method is preferable for conducting exploratory research such as the present study. Additionally, this method can be adapted for use in outside Japan (Scupin, 1997). Following the KJ method, all transcribed data were divided into individual content with a single meaning by three researchers with expertise in sports education or psychology. Next, nearly identical contents were grouped together and labeled as "small categories" for each area (i.e. facilitator and barrier). Three researchers discussed and defined the title of each small cate- 
gory. Then, similar small categories were further grouped into "middle categories". Last, the similarities and differences among the middle categories produced "large categories". Each middle and large category was titled in a way similar to the small category. Initials of facilitators and barriers with identical numbers were added to make discussion easier.

\section{Results}

\section{Characteristics of Participants}

Twenty-five external coaches participated in the interview (Table 1) of which twenty-one were men and four were women. Ages ranged from 22 - 74 years with a mean age of 35.7 years (standard deviation: SD = 17.1). Eleven participants were coaching in junior high school and 14 were coaching in high school. Years of coaching experience ranged from. 5 - 30 with a mean of 6.8 years $(S D=7.9)$. Fifteen external coaches were compensated, and 10 were volunteers.

\section{Facilitators}

Seven large categories of facilitators (i.e. positive feelings, adequate system, positive social support, comfortable climate of SBECSA, environment, growth of external coach, and network building) were grouped (Table 2). Quotes from the study participants are presented below. "Positive feelings" was used to describe enjoyment, parental-like warmth toward team members ("Children are lovely"), the desire to coach, love for the sport, commitment or responsibility ("The relationships with students and their parents have been built. So, I can't break up these relationships"), and a desire to win. "Adequate system" was used to describe little responsibility ("Having no compensation makes me feel better about joining the SBECSA"), compensation, a strong request from an organization ("All graduate students in my laboratory appear to be ordered to coach SBECSA"), and the rights of the external coach ("External coaches can get an ID card, that allows me to watch games near place"). "Positive social support" included a cooperative SBECSA teacher, understanding from the school ("When I go to the SBECSA, teachers seem to be glad. The school principal is also friendly"), understanding from parents, and a cooperative family. "Comfortable climate of SBECSA" involved acceptance of SBECSA ("I want to coach because students want me to join the SBECSA"), growth of team members, and high motivation of team members. "Environment" was used to describe easy access ("Being involved in the SBECSA is easy, because the school is my alma mater", and "The school is near my home"), environment where an external coach can grow ("Working with SBECSA teacher advances my own growth"), and inadequate environment. "Growth of external coach" was composed of growth of external coach, and coaching experience. Finally, "network building" described networking ("I can make a connection with junior high school teachers"), and a positive influence on future career. There were 24 middle categories and 53 small categories with greater detail, as shown in Table 2.

\section{Barriers}

Six large categories of barriers (i.e. negative feelings, inadequate system, lack of support, uncomfortable climate of SBECSA, poor environment, burdens) emerged from the inter-
Table 1.

Demographics of participants and characteristics of SBECSA.

\begin{tabular}{|c|c|c|c|c|c|}
\hline No. & Gender & Age & School & $\begin{array}{l}\text { Extracurricular } \\
\text { activity type }\end{array}$ & Compensation \\
\hline 1 & M & 22 & Junior high & Track and field & Yes \\
\hline 2 & M & 23 & Junior high & Rubber-ball baseball & - \\
\hline 3 & M & 23 & Junior high & Rubber-ball baseball & - \\
\hline 4 & M & 23 & Junior high & Track and field & Yes \\
\hline 5 & M & 23 & Junior high & Basketball & - \\
\hline 6 & M & 23 & High & Rugby & Yes \\
\hline 7 & M & 23 & High & Baseball & - \\
\hline 8 & M & 23 & High & Handball & Yes \\
\hline 9 & M & 24 & High & Soccer & - \\
\hline 10 & M & 27 & High & Soccer & - \\
\hline 11 & M & 27 & High & Archery & Yes \\
\hline 12 & M & 28 & Junior high & Volleyball & Yes \\
\hline 13 & M & 33 & Junior high & Basketball & - \\
\hline 14 & M & 35 & High & Soccer & Yes \\
\hline 15 & M & 36 & Junior high & Badminton & Yes \\
\hline 16 & M & 38 & High & Badminton & - \\
\hline 17 & M & 47 & High & Basketball & Yes \\
\hline 18 & M & 62 & High & Baseball & - \\
\hline 19 & M & 66 & Junior high & Table tennis & Yes \\
\hline 20 & M & 72 & Junior high & Soft tennis & Yes \\
\hline 21 & M & 74 & High & Kendo & Yes \\
\hline 22 & F & 22 & High & Dance & Yes \\
\hline 23 & F & 23 & High & Softball & - \\
\hline 24 & F & 40 & High & Dance & Yes \\
\hline 25 & $\mathrm{~F}$ & 56 & Junior high & Volleyball & Yes \\
\hline
\end{tabular}

views (Table 3). "Negative feelings” included worries about coaching ("I'm bothering with my coaching method"), physical and mental fatigue, concerns about team members becoming injured, and lack of enjoyment. "Inadequate system" described a primitive system ("Mediation system should be more visible", and "Is it difficult to create environment where coaches can learn?”), limitations of the system ("I am sometimes told please don't come to the SBECSA more than twice a week because of a lack of budget”), lack of compensation, and unclear status or role ("I wonder how much should I intervene in SBECSA"). "Lack of support" contained a lack of understanding from the school, uncooperative SBECSA teacher, poor relationships with parents, lack of communication ("It is better to share information about the school with teachers"), opposition from external coaches family, and uncooperative athletic association ("I have many requests for athletic association that I belong to, 


\section{K. AOYAGI ET AL.}

Table 2.

Facilitators of involvement of external coaches.

\begin{tabular}{|c|c|c|}
\hline Large category (7) & Middle category (24) & Small category (53) \\
\hline \multirow{16}{*}{ Positive feelings } & \multirow{6}{*}{ Enjoyment } & f1. sharing a dream with team members \\
\hline & & f2. feeling enjoyment \\
\hline & & f3. having a refreshing change \\
\hline & & f4. achieving a sense of fulfillment \\
\hline & & f5. feeling enjoyment to consider team members \\
\hline & & f6. having fun as a coach \\
\hline & \multirow{3}{*}{$\begin{array}{l}\text { Parental-like warmth toward } \\
\text { team members }\end{array}$} & f7. feeling parental-like warmth toward team members \\
\hline & & f8. desire to continue to involve team members \\
\hline & & f9. desire to help team members continue to engage in SBECSA \\
\hline & \multirow{2}{*}{ Desire to coach } & f10. desire to coach \\
\hline & & f11. desire to have a positive influence as a coach \\
\hline & \multirow{2}{*}{ Love for the sport } & f12. love of the sport that external coach plays \\
\hline & & f13. vitalizing the local sport the external coach plays \\
\hline & \multirow{2}{*}{ Commitment or responsibility } & f14. having a commitment or responsibility \\
\hline & & f15. desire to repay an obligation \\
\hline & Desire to win & f16. desire for team members to win \\
\hline \multirow{5}{*}{ Adequate system } & \multirow{2}{*}{ Little responsibility } & f17. affable status of external coach for team members or their parents \\
\hline & & f18. having little sense of responsibility by getting no compensation \\
\hline & Compensation & f19. getting compensation \\
\hline & Strong request from an organization & f20. being requested by the organization to which external coach belongs \\
\hline & Rights of the external coach & f21. having the right to enter the competition site \\
\hline \multirow{5}{*}{ Positive social support } & \multirow{2}{*}{ Cooperative SBECSA teacher } & f22. having a cooperative SBECSA teacher \\
\hline & & f23. adjusting practice time around external coach's schedule \\
\hline & Understanding from the school & f24. understanding from teachers other than the SBECSA teacher \\
\hline & Understanding from parents & f25. understanding from parents \\
\hline & Cooperative family & f26. support from own family members \\
\hline \multirow{6}{*}{$\begin{array}{l}\text { Comfortable climate } \\
\text { of SBECSA }\end{array}$} & \multirow{3}{*}{ Acceptance of SBECSA } & f27. acceptance of team members \\
\hline & & f28. respect from team members \\
\hline & & f29. team members who take external coach's advice \\
\hline & \multirow{2}{*}{ Growth of team members } & f30. seeing technical improvement in team members \\
\hline & & f31. seeing personal progress in team members \\
\hline & High motivation of team members & f32. high motivation of team members \\
\hline \multirow{7}{*}{ Environment } & \multirow{4}{*}{ Easy access } & f33. alma mater of external coach \\
\hline & & f34. close proximity of school \\
\hline & & f35. being able to go to practice facility using only a commuter pass \\
\hline & & f36. having a coaching environment available \\
\hline & \multirow{2}{*}{$\begin{array}{l}\text { Environment where an external } \\
\text { coach can grow }\end{array}$} & f37. having a SBECSA teacher to learn from \\
\hline & & f38. being on a powerful team \\
\hline & Inadequate environment & f39. being motivated by a poor SBECSA environment \\
\hline
\end{tabular}




\begin{tabular}{|c|c|c|}
\hline \multirow{9}{*}{$\begin{array}{l}\text { Growth of external } \\
\text { coach }\end{array}$} & \multirow{6}{*}{ Growth of external coach } & f40. learning of external coach \\
\hline & & f41. growth of external coach \\
\hline & & f42. enhancement of communication skills \\
\hline & & f43. learning ways to communicate with team members \\
\hline & & f44. enhancing competitive ability of external coach \\
\hline & & f45. enhancing physical fitness of external coach \\
\hline & \multirow{3}{*}{ Coaching experience } & f46. having an educational experience \\
\hline & & f47. accumulating coaching experience \\
\hline & & f48. talking with other coaches or SBECSA teacher \\
\hline \multirow{4}{*}{ Network building } & \multirow{3}{*}{ Networking } & f49. being able to network \\
\hline & & f50. increasing acquaintances \\
\hline & & f51. increasing customers for self-employed external coaches \\
\hline & Positive influence on future career & $\begin{array}{l}\text { f52. perceiving that external coaching is helpful in passing the teacher adaptation examination } \\
\text { f53. getting a position as a part-time teacher }\end{array}$ \\
\hline
\end{tabular}

Note: "f” placed in front of small category means "facilitator". Additionally, each small category was given identical number for discussion.

rather than school"). "Uncomfortable climate of SBECSA" consisted of poor relationships with team members, low motivation of team members, and despair or distress of team members. "Poor environment" involved inconvenient practice time, inconvenient location, inadequate facilities or equipment, and bad weather. Lastly, "burdens" comprised time burdens, pressure or expectations ("Others expect that the SBECSA will become stronger by my coaching, but sometimes it is a burden for me"), and negative effect on primary job. There were 24 middle categories and 54 more detailed small categories, as shown in Table 3.

\section{Discussion}

In the present study, interviews were conducted with 25 external coaches to explore facilitators and barriers associated with their involvement in SBECSA. Numerous facilitators and barriers were identified. The middle category level of barriers included concerns about team members becoming injured, uncooperative SBECSA teacher ("b24. SBECSA teachers who rarely come to the field"), poor relationships with parents, low motivation of team members, inadequate facilities or equipment, time burdens, pressure or expectations ("b52. Unrealistic expectations from teachers or parents”), and a negative effect on primary job. These barriers are consistent with the results of previous quantitative researches that were conducted with external coaches in two prefectural areas (Shioya, 2002; Kanagawa Prefecture Board of Education, 2008; Miyagi Prefecture Board of Education, 2008). The results of the present study suggested the existence of concurrent barriers in other areas of Japan.

Issues and barriers to the recruitment of external coaches found in previous studies conducted with teachers revealed low coaching frequency, lack of compensation, role or responsibility of SBECSA teacher toward external coaches, differences in coaching policies compared with that of external coaches, complicated procedures to involve external coaches, poor relationships with students or parents, and geographical isolation (Iba- raki Prefecture Sports Promotion Council, 2007; Yamagata Prefecture Board of Education, 2010; Williams, Hay, \& Macdonald, 2011). External coaches who participated in the present study also indicated barriers in the middle category level such as limitations of the system ("b13. Institutional limitation on coaching frequency”), lack of compensation, unclear status or role, lack of understanding from the school ("b22. Cumbersome approval procedure to enter the school”), uncooperative SBECSA teacher ("b26. Conflicting opinions with SBECSA teacher”), poor relationships with parents, poor relationships with team members, and inconvenient location. Interestingly, these barriers were reported by both external coaches and teachers. The concurrent perception of these issues indicates that reducing these barriers would affect not only external coaches but also teachers (acceptance side) and would therefore become an effective promoter for the involvement of external coaches. To reduce these barriers, several kinds of support would be valuable. For example, the policy maker could try to increase compensation for external coaches or make procedures easier for entering the school. Furthermore, SBECSA teachers should have increased meetings with external coaches to help build better relationships among students, parents, and external coaches. To address the unclear status or role of external coaches and teachers, some sports associations have defined rules and the rights of external coaches (e.g. do not change practice times without the consent of SBECSA teachers and do not have contact with parents) (Hokkaido Junior High School Physical Culture Association, 2006; Nagano Prefecture Board of Education, 2010). Using these guidelines, it is possible to define clearer roles for external coaches and teachers.

Although some categories were similar to those of previous studies, most facilitators and barriers identified and categorized in the present study, were novel and expressed in more detail. Some categories revealed in the present study suggest that there are many ways to promote the involvement of external coaches in SBECSA. According to a previous questionnaire study, teachers demanded an increase in compensation for external coaches to promote their involvement in SBECSA (Miyagi 
Table 3.

Barriers to involvement of external coaches.

\begin{tabular}{|c|c|c|}
\hline Large category (6) & Middle category (24) & Small category (54) \\
\hline \multirow{7}{*}{ Negative feelings } & \multirow{3}{*}{ Worries about coaching } & b1. gap between a coaching ideal and actual experience \\
\hline & & b2. concerns about own coaching method \\
\hline & & b3. having to engage in tasks other than technical coaching \\
\hline & \multirow{2}{*}{ Physical and mental fatigue } & b4. feeling fatigued \\
\hline & & b5. poor competition score for external coach \\
\hline & Concerns about team members becoming injured & b6. concerns about team members becoming injured \\
\hline & Lack of enjoyment & b7. not enjoying the coaching experience \\
\hline \multirow{12}{*}{ Inadequate system } & \multirow{5}{*}{ Primitive system } & b8. no mediation system for external coaches \\
\hline & & b9. no credit on teacher adaptation examination \\
\hline & & b10. no educational system for external coaches \\
\hline & & b11. no environment where coaches can work \\
\hline & & b12. not continuing the involvement of external coaches \\
\hline & \multirow{3}{*}{ Limitations of the system } & b13. institutional limitation on coaching frequency \\
\hline & & b14. institutional limitation on working range \\
\hline & & b15. institutional limitation on number of external coaches \\
\hline & \multirow{2}{*}{ Lack of compensation } & b16. little or no compensation \\
\hline & & b17. not an hourly wage \\
\hline & \multirow{2}{*}{ Unclear status or role } & b18. unsure of status or role \\
\hline & & b19. no official request from school \\
\hline \multirow{16}{*}{ Lack of support } & \multirow{4}{*}{ Lack of understanding from the school } & b20. school policy that prohibits involvement of external coaches \\
\hline & & b21. teachers who have negative opinions about involvement of external coaches \\
\hline & & b22. cumbersome approval procedure to enter the school \\
\hline & & b23. being unrecognized by students (excluding team members) \\
\hline & \multirow{4}{*}{ Uncooperative SBECSA teacher } & b24. SBECSA teachers who rarely come to the field \\
\hline & & b25. insufficient efforts to accept external coaches \\
\hline & & b26. conflicting opinions with SBECSA teacher \\
\hline & & b27. insufficient contact with SBECSA teacher \\
\hline & \multirow{4}{*}{ Poor relationships with parents } & b28. lack of understanding from parents \\
\hline & & b29. poor relationships with parents \\
\hline & & b30. insufficient parenting \\
\hline & & b31. poor relationships between parents and SBECSA teacher \\
\hline & \multirow{2}{*}{ Lack of communication } & b32. no chance for information exchange \\
\hline & & b33. no interaction among coaches \\
\hline & Opposition from external coaches family & b34. lack of support from external coaches family members \\
\hline & Uncooperative athletic association & b35. uncooperative athletic association \\
\hline \multirow{5}{*}{$\begin{array}{l}\text { Uncomfortable } \\
\text { climate of SBECSA }\end{array}$} & \multirow{3}{*}{ Poor relationships with team members } & b36. poor relationships with team members \\
\hline & & b37. having team members who do not accept external coach’s instruction \\
\hline & & b38. retirement of external coach's own child from SBECSA \\
\hline & Low motivation of team members & b39. low motivation of team members \\
\hline & Despair or distress of team members & b40. despair or distress of team members \\
\hline
\end{tabular}




\begin{tabular}{|c|c|c|}
\hline \multirow{4}{*}{ Poor environment } & Inconvenient practice time & b41. inconvenient practice time \\
\hline & Inconvenient location & b42. long distance to school \\
\hline & Inadequate facilities or equipment & b43. inadequate facilities or equipment \\
\hline & Bad weather & b44. bad weather \\
\hline \multirow{10}{*}{ Burdens } & \multirow{4}{*}{ Time burdens } & b45. decrease in private time \\
\hline & & b46. requires time \\
\hline & & b47. no extra time to do part-time job \\
\hline & & b48. having to sacrifice holidays \\
\hline & \multirow{4}{*}{ Pressure or expectations } & b49. having the responsibility \\
\hline & & b50. not finishing coaching obligations before the next coaching day \\
\hline & & b51. pressure from alumni organization \\
\hline & & b52. unrealistic expectations from teachers or parents \\
\hline & \multirow{2}{*}{ Negative effect on primary job } & b53. difficulty balancing primary job and coaching \\
\hline & & b54. having to compete against other school teams \\
\hline
\end{tabular}

Note: "b" placed in front of small category means "barrier". Additionally, each small category was given identical number for discussion.

Prefecture Board of Education, 2008). Williams et al. (2011) also indicated that prohibitive costs were one reason for not outsourcing coaches. However, some external coaches in the present study indicated that having little or no compensation was a facilitator in the adequate system category ("f18. Having little sense of responsibility by getting no compensation”). Additionally, 10 of the participants in the present study were coaching in SBECSA, even though they did not receive any compensation. Furthermore, several categories considered facilitators that represented non-monetary compensation (" $\mathrm{f} 4$. Achieving a sense of fulfillment" in the positive feelings category, "f49. Being able to network", and "f52. Perceiving that external coaching is helpful in passing the teacher adaptation examination" in the network building category). Therefore, monetary compensation might not be the only purpose for engaging as an external coach. Tomioka (1993) suggested that compensation or reward from working included a sense of fulfillment, enjoyment, and having good colleagues. Thus, the promotion of external coaches in SBECSA may require expanding opportunities for networking and including external coaching experience in the evaluation criteria of teacher adaptation examinations as well as providing compensation. Accordingly, Saitama prefecture in Japan listed volunteer activity as an evaluation criterion for teacher adaptation examinations (Saitama Prefecture Board of Education, 2011). External coaching in SBECSA with little or no compensation could be considered a volunteer activity. Thus, it is realistic and easily achievable to state clearly that external coaching is a volunteer activity in the teacher adaptation examination guidebook. Modification of evaluation criteria in the teacher adaptation examination could be an efficient promotional strategy for attracting external coaches who want to be teachers.

As limitations of the system, "b13. Institutional limitation on coaching frequency" and "b15. Institutional limitation on number of external coaches" were revealed as noteworthy barriers. Similar limitations were interpreted in a project by MEXT, where the number of external coaches was limited to 50, and coaching frequency was limited to 25 times a year (i.e. ap- proximately twice a month) (Kochi Prefecture Board of Education, 2011). Therefore, it is critical to reduce or abolish institutional limitations on coaching frequency to promote the involvement of external coaches for SBECSA.

The involvement of external coaches can reduce the burden of SBECSA teachers in relation to attending SBECSA (Japan Senior High School Teachers and Staff Union, 2008; Tokyo Metropolitan Board of Education, 2008). However, "b24. SBECSA teachers who rarely come to the field" revealed that uncooperative SBECSA teachers are a barrier. External coaches requested that SBECSA teachers attend SBECSA more often. The Tokyo Metropolitan Board of Education (2008) suggested that SBECSA teachers should engage in the SBECSA and share roles of coaching and management with external coaches. To address this barrier, cooperation between teachers and external coaches is essential.

There were two conflicting views regarding compensation and inadequate facilities in the present study. First, "f18. Having little sense of responsibility by getting no compensation" and "f19. Getting compensation" were considered facilitators by some external coaches. Second, "f39. Being motivated by a poor SBECSA environment” was considered a facilitator, and "b43. Inadequate facilities or equipment" was deemed a barrier. From these conflicting views, it is unclear whether increasing compensation and improving the environment would be a facilitator or barrier to promoting the involvement of external coaches. The present study only clarified the contents of facilitators and barriers, and their classifications. The influence of each facilitator and barrier on external coaches' involvement in SBECSA related to their sociodemographics and characteristics of SBECSA remain unclear. Thus, it needs to consider different perceptions of categories by individuals with different sociodemographics and characteristics of SBECSA in future studies. Although there were some limitations in the present study, clarification of various and detailed facilitators and barriers is of value for future studies and the further promotion of SBECSA. A wide range of sociodemographic characteristics of participants helped to collect varied facilitators and barriers. 
The SBECSA environment could be made more efficient by enhancing facilitators and removing barriers identified by external coaches.

\section{Conclusion}

In conclusion, the present study identified multiple facilitators and barriers associated with external coach involvement in SBECSA. Providing opportunities for external coaches to network, specifying external coaching experience as meeting an evaluation criterion on the teacher adaptation examination and increasing the number of meetings between teachers and external coaches are important, as well as increasing monetary compensation. Additionally, reducing or removing institutional limitations and increasing cooperation between teachers and external coaches will hopefully promote the involvement of external coaches in SBECSA. These findings may help schools and policy makers in discussions regarding appropriate SBECSA, which would further enhance the attraction of SBECSA for students, teachers, and external coaches.

\section{Acknowledgements}

The authors would like to thank all participating external coaches, and the peers and teachers who introduced participants. The present study was supported by the Sasakawa Sports Research Grant from Sasakawa Sports Foundation, and Global COE Program "Sport Sciences for the Promotion of Active Life" from the Ministry of Education, Culture, Sports, Science and Technology in Japan.

\section{REFERENCES}

All Japan High School Athletic Federation (2012). News from head office. http://www.zen-koutairen.com/f_publish.html

Australian Bureau of Statistics (2012). Children's participation in cultural and leisure activities.

http://www.abs.gov.au/ausstats/abs@.nsf/mf/4901.0

Barnett, L. A. (2007). "Winners" and "Losers": The effects of being allowed or denied entry into competitive extracurricular activities. Journal of Leisure Research, 39, 316-344.

Cote, J., \& Gilbert, W. (2009). An integrative definition of coaching effectiveness and expertise. International Journal of Sports Science and Coaching, 4, 307-322. doi:10.1260/174795409789623892

Dotterer, A. M., McHale, S. M., \& Crouter, A. C. (2007). Implications of out-of-school activities for school engagement in African American adolescents. Journal of Youth and Adolescence, 36, 391-401. doi:10.1007/s10964-006-9161-3

Edwards, M. B., Kanters, M. A., \& Bocarro, J. N. (2011). Opportunities for extracurricular physical activity in North Carolina middle schools. Journal of Physical Activity and Health, 8, 597-605.

Flintoff, A. (2008). Targeting Mr average: Participation, gender equity and school sport partnerships. Sport, Education and Society, 13, 393411. doi:10.1080/13573320802445017

Fredricks, J. A., \& Eccles, J. S. (2006). Is extracurricular participation associated with beneficial outcomes? Concurrent and longitudinal relations. Developmental Psychology, 42, 698-713.

doi:10.1037/0012-1649.42.4.698

Griggs, G. (2010). For sale-Primary physical education. $£ 20$ per hour or nearest offer. Education 3-13, 38, 39-46. doi:10.1080/03004270903099793

Hokkaido Junior High School Physical Culture Association (2006). As regards external coach in Hokkaido junior high school athletic meeting.

Ibaraki Prefecture Sports Promotion Council (2007). The way of future school-based extracurricular sports activity. http://www.edu.pref.ibaraki.jp/board/bunspo/sports/setti/toushin.pdf Japan Senior High School Teachers and Staff Union (2008). Final report of actual condition survey for issues of school-based extracurricular sports activity in 2006.

Kanagawa Prefecture Board of Education (2008). Research report for sports activity of secondary school student. http://www.pref.kanagawa.jp/uploaded/attachment/176796.pdf

Kawakita, J. (2004). Zoku hassouhou. Tokyo: Chuokoron-Shinsha, Inc. Kochi Prefecture Board of Education (2011). Implementation guidance of practice support project promoting local sport human resources.

LaVoi, N. M., \& Dutove, J. K. (2012). Barriers and supports for female coaches: An ecological model. Sports Coaching Review, 1, 17-37. doi:10.1080/21640629.2012.695891

Lipscomb, S. (2007). Secondary school extracurricular involvement and academic achievement: A fixed effects approach. Economics of Education Review, 26, 463-472. doi:10.1016/j.econedurev.2006.02.006

Ministry of Education, Culture, Sports, Science and Technology in Japan (1997). Report of investigative research for way of school-based extracurricular sports activity.

http://www.mext.go.jp/b_menu/shingi/chousa/sports/001/toushin/971 201.htm

Ministry of Education, Culture, Sports, Science and Technology in Japan (2008). The course of study in junior high school. http://www.mext.go.jp/a_menu/shotou/new-cs/youryou/1304424.htm

Ministry of Education, Culture, Sports, Science and Technology in Japan (2009a). The course of study in higher school. http://www.mext.go.jp/a_menu/shotou/new-cs/youryou/1304427.htm

Ministry of Education, Culture, Sports, Science and Technology in Japan (2009b). White paper on education, culture, sports, science and technology.

http://www.mext.go.jp/b_menu/hakusho/html/hpab200901/1295623. htm

Ministry of Education, Culture, Sports, Science and Technology in Japan (2010). Sport-oriented nation strategy: Sport community Japan. http://www.mext.go.jp/a_menu/sports/rikkoku/1297182.htm

Ministry of Education, Culture, Sports, Science and Technology in Japan (2011). Basic law of sports. http://www.mext.go.jp/a_menu/sports/kihonhou/attach/1307658.htm

Ministry of Education, Culture, Sports, Science and Technology in Japan (2012). National survey result of physical and athletic capacity. http://www.mext.go.jp/b_menu/toukei/chousa04/tairyoku/kekka/k_d etail/1326589.htm

Miyagi Prefecture Board of Education (2008). Research for schoolbased extracurricular sports activity of secondary school.

Nagano Prefecture Board of Education (2010). Companion of schoolbased extracurricular sports activity.

Nakazawa, A. (2011). A postwar history of extracurricular sports activities in Japan (1): Focusing on the transition of the actual situation and policy. Hitotsubashi Bulletin of Social Sciences, 3, 25-46.

Nippon Junior High School Physical Culture Association (2012). Spread sheet of research for number of member school and student. http://www18.ocn.ne.jp/ njpa/kamei.html

Nishijima, H., Yano, H., \& Nakazawa, A. (2007). A sociological study of coaching and management of club activities in junior high schools: Based on a questionnaire survey to teachers of sports club activities in two prefectures and Tokyo metropolitan. Bulletin of Faculty of Education in the University of Tokyo, 47, 101-130.

Saitama Prefecture Board of Education (2011). Selection policy of first high school teacher adaptation exam in 2011's Saitama prefecture.

Sasakawa Sports Foundation (2011). Installation of sports leader bank in each prefecture. Sports White Paper: Future That Sports Should to Aspire, 86-88.

Schaefer, D. R., Simpkins, S. D., Vest, A. E., \& Price, C. D. (2011). The contribution of extracurricular activities to adolescent friendships: New insights through social network. Developmental Psychology, 47, 1141-1152. doi:10.1037/a0024091

School-Based Extracurricular Sports Activity in Junior High School “Nagano Model” Exploratory Committee (2004). Proposal of schoolbased extracurricular sports activity in junior high school "Nagano model". 


\section{K. AOYAGI ET AL.}

http://www.pref.nagano.lg.jp/kyouiku/taiiku/bukatu/teigen/teigen.pdf Scupin, R. (1997). The KJ method: A technique for analyzing data derived from Japanese ethnology. Human Organization, 56, 233-237. Shernoff, J. D., \& Vandell, L. D. (2007). Engagement in after-school program activities: Quality of experience from the perspective of participants. Journal of Youth and Adolescence, 36, 891-903. doi:10.1007/s10964-007-9183-5

Shioya, K. (2002). External coach and volunteer in school-based extracurricular sports activity. Journal of Health, Physical Education and Recreation, 52, 285-289.

Sport Council Wales (2009). Young people's participation in sport. Sportsupdate, 62, 11-16.

Stewart, V., Lindsay, O., \& Trevor, C. (2011). The role of the coach in facilitating positive youth development: moving from theory to practice. Journal of Applied Sport Psychology, 23, 33-48. doi:10.1080/10413200.2010.511423
Tokyo Metropolitan Board of Education (2008). Companion of coaching school-based extracurricular activity for external coach.

http://www.kyoiku.metro.tokyo.jp/press/bukatsu_tebiki.pdf

Tomioka, A. (1993). Organization and human behavior. Tokyo: Hakuto-shobo, Inc.

Whiteley, R. F., \& Richard, G. (2012). Timetabling and extracurricular activities: A study of teachers' attitudes towards preparation time. Management in Education, 26, 6-12. doi:10.1177/0892020611426894

Williams, J. B., Hay, J. P., \& Macdonald, D. (2011). The outsourcing of health, sport and physical educational work: A state of play. Physical Education and Sport Pedagogy, 16, 399-415. doi:10.1080/17408989.2011.582492

Yamagata Prefecture Board of Education (2010). As regards a way of future school-based extracurricular sports activity.

http://www.pref.yamagata.jp/ou/kyoiku/700021/21unndoubukatudou arikata.pdf 